\title{
Demonstrating Fast Current Measurement from Femtoamperes to Nanoamperes with an Ultra-Low Noise Current Amplifier
}

\author{
Cornelius Wendt, Alexander Bohnhorst, Stefan Zimmermann and Ansgar T. Kirk

\begin{abstract}
Leibniz University Hannover, Institute of Electrical Engineering and Measurement Technology, Department of Sensors and Measurement Technology, Appelstr. 9A, 30167 Hannover, Germany

Contact: wendt@geml.uni-hannover.de
\end{abstract}

\section{Introduction}

In a large number of sensor principles, the physical or chemical property being analysed is converted into an electric current, a charge, a resistance or a capacitance, whereby in the last two cases an electric current or a charge needs to be measured. Especially ionization-based sensors such as photoionization detectors (PID) or flame ionization detectors (FID) generating currents ranging from femto- to nanoamperes. In all these cases, a current amplifier converting the electric current into a measurable voltage forms the link between the sensor and the rest of the measurement system.

The performance of this current amplifier has a decisive influence on the performance of the entire measurement system. For low limits of detection, the current amplifier must offer low noise, for a large linear range large dynamic range, and for short response times high bandwidth. The combination of all these properties in a single current amplifier is a major challenge.

\section{Experimental}
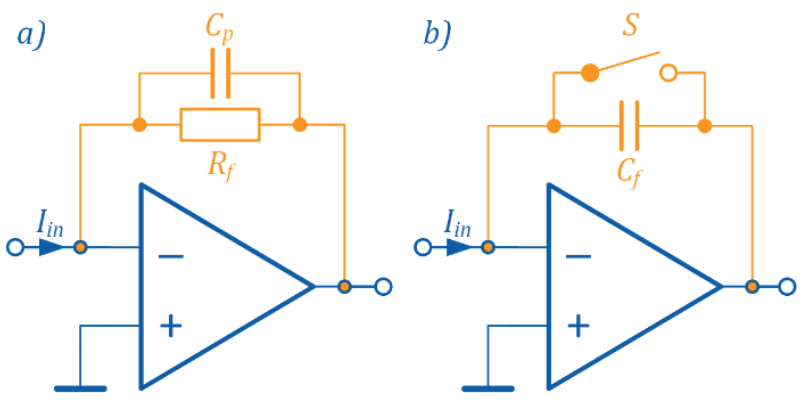

Figure 1: a) Resistive transimpedance amplifier with feedback resistor $R_{f}$ and parasitic capacitance $C_{p}$. b) Capacitive transimpedance amplifier with feedback capacitance $C_{f}$ and reset switch $S$.

Usually, the input stage of a current amplifier is designed as a resistive transimpedance amplifier, i.e. the current to be measured is converted into a voltage via a resistor. This is achieved using the circuit in Figure $1 \mathrm{a}$ ), in which the operational amplifier regulates the output voltage so that the current to be measured flows through the parallel network of the feedback resistor $R_{f}$ and its parasitic capacitance $C_{p}$.

The higher the resistance of $R_{f}$, the lower the noise current standard deviation $\sigma_{i}$ caused by the thermal or Johnson-Nyquist noise as given by eq. $1[1,2]$. It depends on the Boltzmann constant $\mathrm{k}_{\mathrm{b}}$, the absolute temperature $T$ and and the noise bandwidth, which is approximated by the amplifier bandwidth $f_{g}$ when assuming an ideal filter.

$$
\sigma_{i}=\sqrt{\frac{4 \mathrm{k}_{\mathrm{b}} T f_{g}}{R_{f}}}
$$

Thus, to reduce amplifier noise at a given temperature and amplifier bandwidth, the value of the feedback resistor $R_{f}$ should be chosen as high as possible. At the same time, however, a higher resistance reduces the maximum measurable current at a given output voltage and the maximum bandwidth at a given parasitic capacitance. On the one hand, as the noise current only reduces with the square root of the resistance, but the maximum measurable current reduces linearly, extremely high output voltages become necessary to achieve a large dynamic range. On the other hand, extremely low parasitic capacitances or complex compensation schemes become necessary to achieve a high bandwidth.

An alternative to overcome these limitations are capacitive transimpedance amplifiers as shown in Figure $1 \mathrm{~b}$ ), which integrate the current to be measured on a feedback capacitor $C_{f}$ and differentiate the signal again after digitization. Noise-wise, this is equivalent to a nearly infinite resistance, but the maximum measurable current can now be increased by resetting the capacitor more often through the reset switch S. However, leakage currents and charge injection of the reset switch result in new sources of error, limiting the performance of this otherwise superior concept. Here, we present an active reset architecture, which actively regulates the voltage across the capacitor to zero during reset [3], successfully compensating the errors introduced by the switches. This enables the design of a current amplifier with a unique combination of low noise, large dynamic range and high bandwidth. The basic setup is shown in Figure 2. 


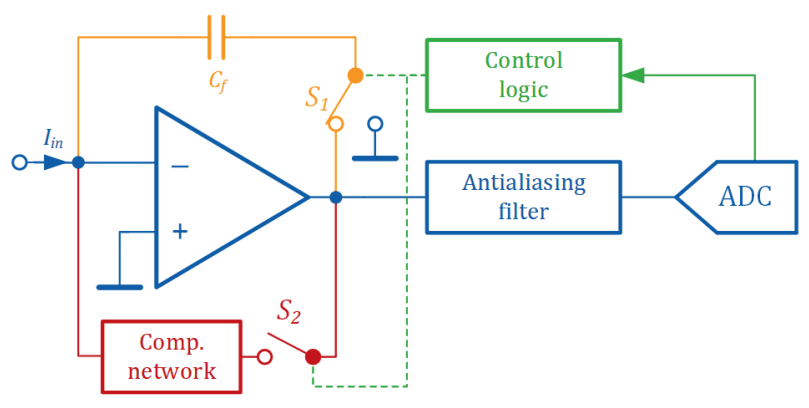

Figure 2: Basic scheme of a capacitive transimpedance amplifier with the active reset architecture, an antialiasing filter and the analog-to-digital conversion

The major advantage becomes obvious when studying the equivalent circuits of the measurement and reset configuration as shown in Figure 3. In the measurement configuration, the circuit maximizes the open resistance of the critical switch $S_{2}$ through a currentlimiting compensation network in series, prevent leakage current and charge injection. Consequently, the circuit acts like an almost ideal integrator, whereby the resulting gain after differentiation only depends on the size of the capacitor $C_{f}$. When a certain charge has been integrated, the output voltage of the operational amplifier approaches its threshold, and the circuit is set to the reset configuration by the control logic. In this configuration, the closed switch $S_{2}$ turns the operational amplifier into a simple buffer, while the integration capacitor is connected between the inverting input and ground by switch $S_{1}$. Since one side of the integration capacitor and the non-inverting input of the operational amplifier are connected to ground, the voltage across the capacitor is regulated to $0 \mathrm{~V}$ the capacitor is actively discharged in a quick and reliable fashion by the operational amplifier.

\section{Measurement}
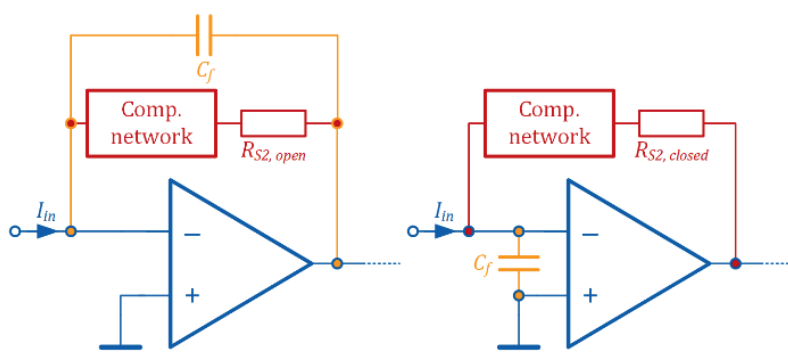

Figure 3: Equivalent circuit of the capacitive transimpedance amplifier with the active reset architecture in measurement configuration (left) and in reset configuration (right). Only the resistance of $S_{2}$ is shown, as $S_{1}$ is not connected to any critical nodes of the circuit.
This design has been successfully implemented in the demonstrator shown in Figure 4. Capacitive transimpedance amplifier, antialiasing filter and ADC are supplied galvanically isolated from earth in order to suppress unwanted interference signals. A microcontroller serves as control logic and USB interface to the measurement computer. It receives the digitized samples of the ADC and, if necessary, triggers the reset of the integration capacitor. The data are oversampled with a frequency of $1 \mathrm{kHz}$, which reduces the requirements on the digital filters.

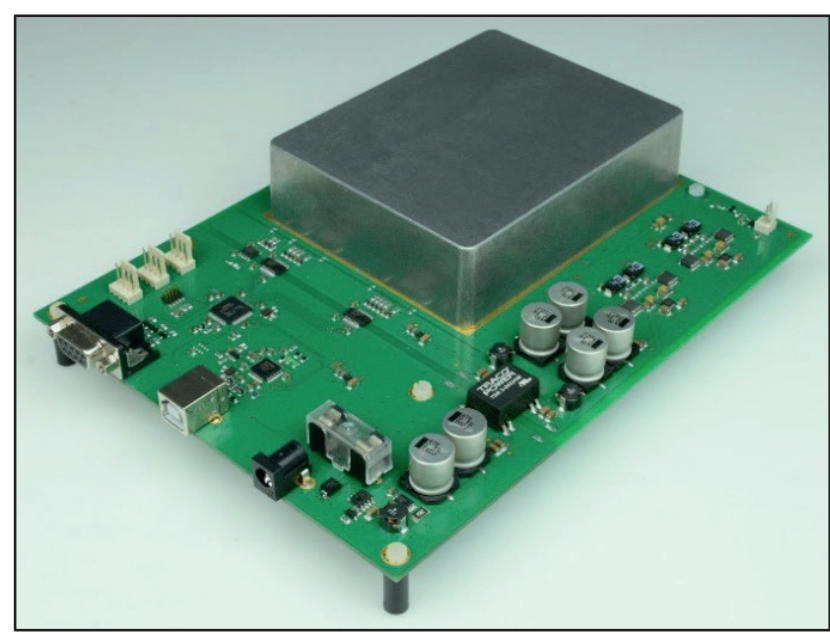

Figure 4: Photo of the capacitive current amplifier. The transimpedance amplifier circuit is covered by a shield to prevent electromagnetic interference.

\section{Results and Discussion}

The developed capacitive transimpedance amplifier shown in Figure 4 achieves a standard deviation of the measured current of $3.4 \mathrm{fA}$ at a $3 \mathrm{~dB}$ bandwidth of $48 \mathrm{~Hz}$, which corresponds to a noise current density of just $0.49 \mathrm{fA} / \sqrt{\mathrm{Hz}}$ assuming a uniform distribution over the frequency spectrum. As shown by the comparison in Figure 5, this value is only $20 \%$ above the theoretically possible noise current density of an ideal resistor with a resistance of $100 \mathrm{G} \Omega,(0.41 \mathrm{fA} / \sqrt{\mathrm{Hz}})$.

However, in a real amplifier, the noise from the operational amplifier and additional noise sources of the resistor beyond Johnson-Nyquist noise would add to this. At the same time, such a high resistance as feedback element in a transimpedance amplifier would already be completely impractical for many realistic applications. On the one hand, the parasitic capacitance of the circuit would need to be just $33 \mathrm{fF}$ to achieve the bandwidth of $48 \mathrm{~Hz}$ achieved with the capacitive transimpedance amplifier. On the other hand, to amplify a current of $10 \mathrm{nA}$ at a resistor value of $100 \mathrm{G} \Omega$, an output voltage of $1 \mathrm{kV}$ would already be required. 


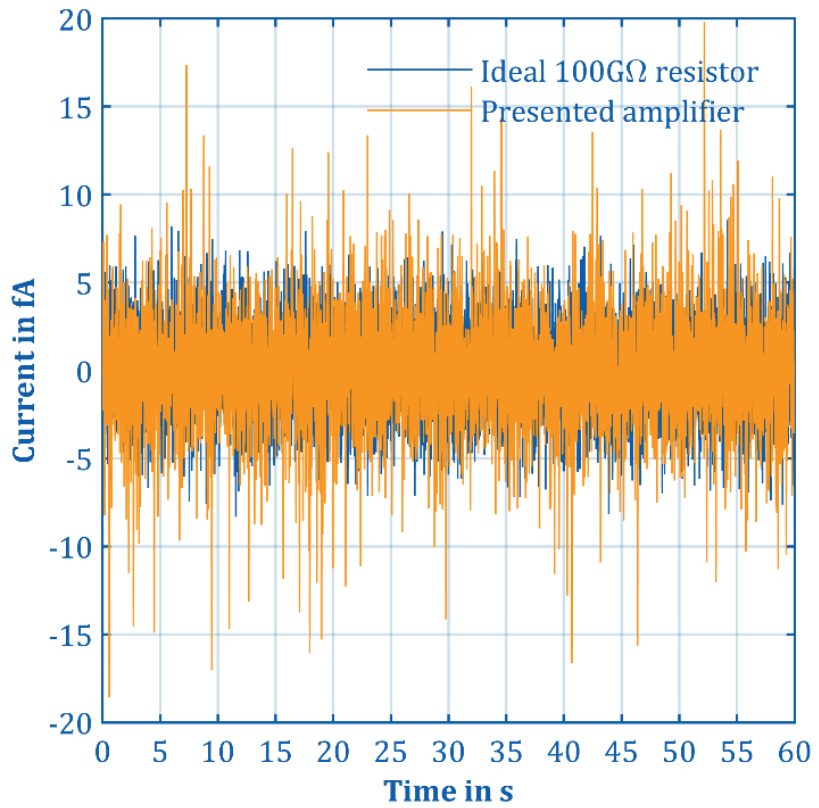

Figure 5: Comparison of the noise current of the developed capacitive transimpedance amplifier with the thermal noise of an ideal $100 \mathrm{G} \Omega$ resistor. Both traces use the same filter.

In contrast, the capacitive transimpedance amplifier demonstrator shown here runs with a conventional operational amplifier and is capable of covering a measurement range of six orders of magnitude from $10 \mathrm{fA}$ to $10 \mathrm{nA}$ without any range switching (Figure 6) while achieving the mentioned low noise current density of $0.49 \mathrm{fA} / \sqrt{\mathrm{Hz}}$.

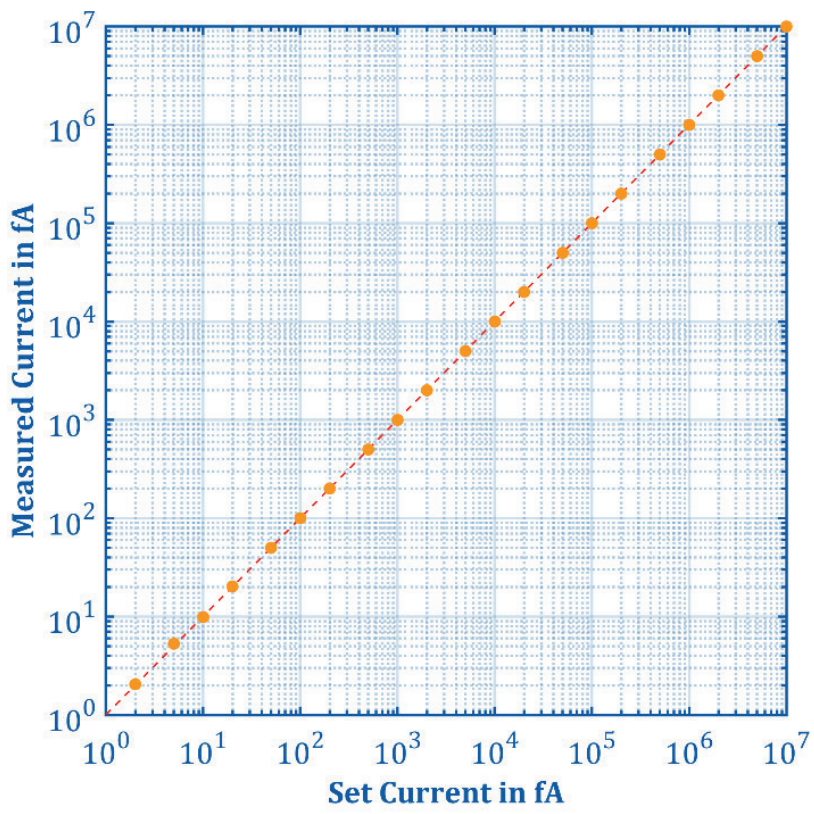

Figure 6: Current measured (dots) with the capacitive transimpedance amplifier against the set value of a current source from Keithley Instruments and Keysight Technologies and linear regression (dashed line, $\mathrm{R}^{2}=0.99$ ).

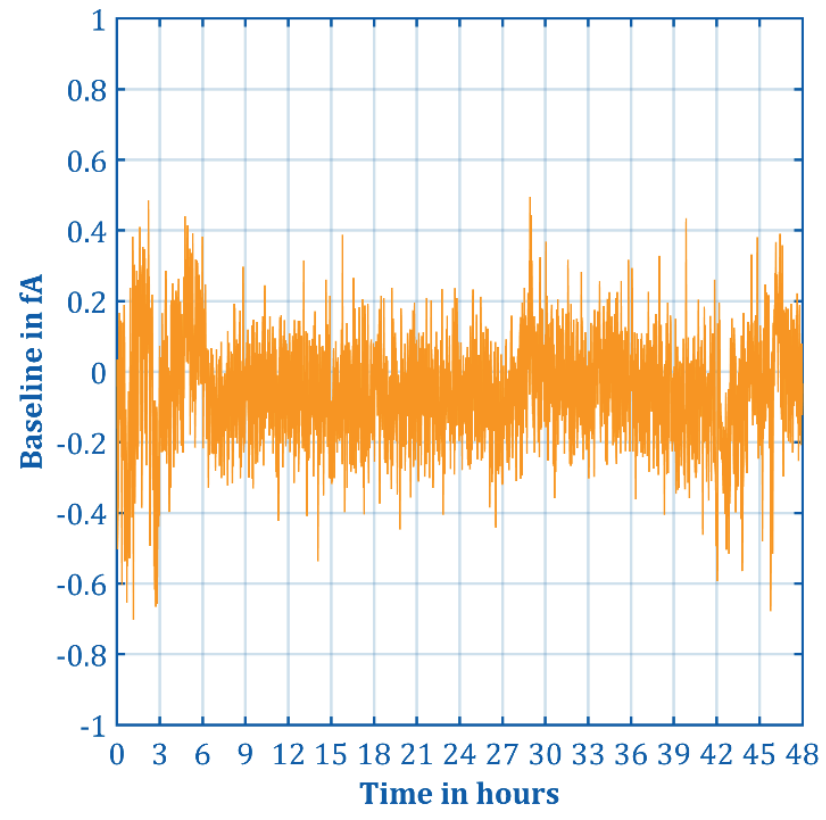

Figure 7: Baseline stability of the developed capacitive transimpedance amplifier. The baseline was determined using the median over five second measurement intervals each.

Moreover, the demonstrator achieves excellent zero-point stability even without temperature control. Over a period of two days, the zero point remained within \pm 500 aA as shown in Figure 7 .

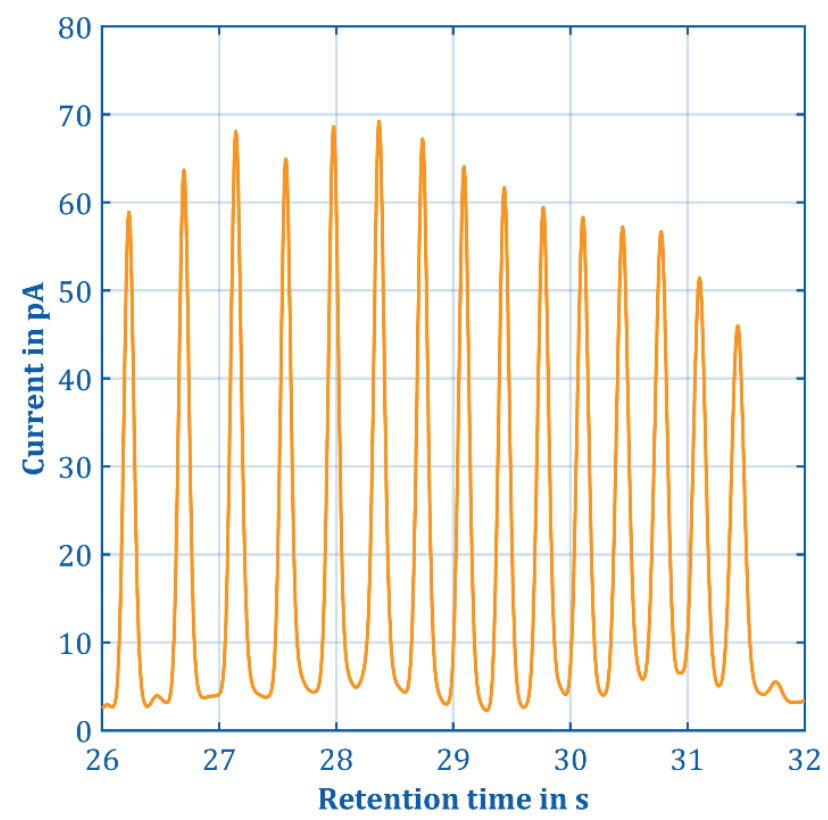

Figure 8: Measurement of current pulses generated by a flame ionization detector (FID) during a gas chromatograph (GC) run.

Figure 8 shows an exemplary measurement of fast current pulses emanating from a flame ionization de- 
tector (FID) coupled to a hyper-fast gas chromatograph (GC) [4]. In this application, the characteristics of the presented amplifier are of particular advantage. The linear output signal of the FID can cover up to 7 orders of magnitude, while the hyper-fast GC generates current pulses with a full width at half maximum (FWHM) of less than $100 \mathrm{~ms}$, thus requiring a high bandwidth for sampling.

\section{Conclusion}

In this work, we presented a new design of a capacitive transimpedance amplifier. As is usual for capacitive transimpedance amplifiers, it is characterised by a very low current noise density. The active reset architecture with two switches in the feedback loop of the operational amplifier and the combination of analogue and digital filters enables a continuous measurement of the current with wide linear range, excellent zero-point stability and high bandwidth.

\section{Acknowledgements}

The project "Femtoampere $(\mathrm{fA})$ bis Mikroampere $(\mu \mathrm{A})$ schnell erfassen - FUSE" is funded by the German Federal Ministry for Economic Affairs and Energy and the European Social Fund as part of the EXIST program.

\section{References}

[1] J.B. Johnson, Thermal Agitation of Electricity in Conductors, Phys. Rev. 32 (1928) 97-109.

[2] H. Nyquist, Thermal Agitation of Electric Charge in Conductors, Phys. Rev. 32 (1928) 110-113.

[3] A.T. Kirk, C. Wendt, S. Zimmermann, Rücksetzschaltung für einen kapazitiven Transimpedanzverstärker, PCT/EP 2019077364 A1, 2019.

[4] P. Boeker, J. Leppert, Flow Field Thermal Gradient Gas Chromatography, Anal. Chem. 87 (2015) 9033-9041. 